\title{
(Not So) Dangerous Liaisons: A Framework for Evaluating Collaborative Research Projects
}

\author{
Pinar Oztop"\#* (D) Frank Loesche\# (iD , Diego S. Maranan\# (iD) Kathryn B. Francis" \\ Vaibhav Tyagi iD , \& Ilaria Torre \\ CogNovo \\ Plymouth University, UK \\ \# Authors with equal contribution \\ *Corresponding author Pinar.Oztop@CogNovo.eu
}

Received 13 May 2017; accepted 30 September 2017; published 21 November 2017.

\begin{abstract}
With advances in research environments and the accompanying increase in the complexity of research projects, the range of skills required to carry out research calls for an increase in interdisciplinary and collaborative work. CogNovo, a doctoral training program for $25 \mathrm{PhD}$ students, provided a unique opportunity to observe and analyze collaborative processes. We propose a process-oriented framework for understanding research collaborations along two dimensions: interpersonal and project-related. To illustrate the utility of this process-oriented framework, we apply the framework matrix to several collaborations that emerged within the CogNovo program. The framework that we introduce has several advantages over existing metrics. Firstly, we offer a process-oriented-as opposed to product-oriented-evaluation of interdisciplinary and collaborative endeavors. Secondly, we propose a means of assessment that preserves the distinctive profile (or "fingerprint") of a given collaborative project, thus capturing the uniqueness of each project and its environment.
\end{abstract}

Keywords: collaboration fingerprint; collaborative framework; group work; interdisciplinary research; organizational team performance; research assessment. 
With achievements increasingly arising from teamwork, "collaboration" has acquired a vital role in organizational, educational, and research contexts (Larivière, Gingras, Sugimoto, \& Tsou, 2014). In particular, research collaboration has received increased attention, with many leading institutions arguing that complex contemporary issues (such as health, environment, and mobility) require solutions that combine insights from different disciplines (National Academies, 2005; as cited in van Rijnsoever \& Hessels, 2011). The complex nature of these issues increasingly necessitates that knowledge and solutions can be combined from multiple disciplines (Buanes \& Jentoft, 2009). Research collaboration has been described in various contexts and by various approaches, with a lack of consensus over its definition; this is why it is often defined under the umbrella term "collaboration" (Bukvova, 2010). What "interdisciplinary collaboration" entails has remained particularly unclear (Huutoniemi, Klein, Bruun, \& Hukkinen, 2010).

Nevertheless, a common theme among various collaborations is that they involve engagement and interaction between two or more people at one time or repeatedly, in order to achieve a common goal (Patel, Pettitt, \& Wilson, 2012). Identifying which factors constitute "successful" interdisciplinary, multidisciplinary, or transdisciplinary ${ }^{1}$ collaboration and what participating members can do to nurture these is "of significant theoretical interest" (Mansilla, Boix, Lamont, \& Sato, 2012, p. 2). Beyond theory, shedding light on this "black box" (Kurtzberg \& Amabile, 2001) has also become an increasing priority for funding bodies and research in industry (Mansilla et al., 2012).

How to operationalize research collaboration is a topic of debate (Katz \& Martin, 1997). Various approaches have been adopted in order to evaluate research collaborations, including bibliometrics, interviews, observations, experiments, and social network analysis (Groboljšek, Ferligoj, Mali, Kronegger, \& Iglič, 2014). Measuring publications through co-authorship evaluation, where publications become the ultimate indicator for collaboration success, is particularly common (Bukvova, 2010). More specifically, the mean number of authors per paper (termed the "Collaborative Index", Lawani, 1980; as cited in Savanur \& Srikanth, 2010), the proportion of multiauthored papers (termed the "Degree of Collaboration", Subramanyam, 1983), or a combination of these (termed the "Collaboration Coefficient"; Ajiferuke, Burrel, \& Tague, 1988) have been used as metrics to assess the scope of collaboration across fields or disciplines (Savanur \& Srikanth, 2010). However, an important point that is often overlooked is that not all research collaborations result in co-authored publications, nor are all co-authorships born out of collaborations (Bukvova, 2010).

\footnotetext{
${ }^{1}$ Although often used interchangeably or without clear definition (Lawrence, 2010), here we adopt the following definitions. Interdisciplinarity "unites" and "synthesises" links between disciplines to form a "coherent whole," multi-disciplinarity draws on information from multiple disciplines but stays within disciplinary limits, and transdisciplinarity brings disciplines together in new contexts and transcends existing disciplinary boundaries (Choi \& Pak, 2006, p. 351). Of course, these categories are not always mutually exclusive given the complexity of many research projects (Klein, 2008).
} 
In addition, the publication of interdisciplinary research appears to be more difficult, resulting in a lower number of interdisciplinary publications and co-authorships. Bruce, Lyall, Tait, and Williams (2004) identify the lack of opportunities to publish interdisciplinary results in high-ranking journals as a discouraging factor to work on interdisciplinary topics.

Evaluating collaborations using product-based approaches, in which outputs of collaborations (i.e., co-authorships) are accepted as indicators of collaboration success, has the advantage of using easily accessible and measurable data (see Groboljšek et al., 2014, for a review). However, these approaches often undervalue the importance of the collaboration process. In their literature review, Aboelela et al. (2007) explored the different views on interdisciplinarity in order to compose a theoretical definition of interdisciplinary research. Key components of interdisciplinary research from the literature included: covering qualitatively different research disciplines; creating a continuum of collaboration which varies from brief communications to mutual integration; establishing a platform for cooperation, interaction, communication, and sharing. In fact, this latter component is considered critical in the majority of interdisciplinarity definitions (Aboelela et al., 2007). As such, a process-based framework, which focuses on what Callard and Fitzgerald call the "choreography" (2015, p. 80) of cooperation and integration between group members, could offer valuable insights for understanding and evaluating research collaborations. Therefore, while we cannot deny the value of collaborative outputs, in the present paper, we focus on the process of collaboration and the dynamics of interdisciplinary integration. We interpret examples of collaborations within the same organization on two dimensions: interpersonal and project-based. To capture and evaluate these collaborations, we propose a process-focused matrix. We present several example studies of collaborations that were fostered within the interdisciplinary CogNovo project ${ }^{2}$ (Maranan, Loesche, \& Denham, 2015), and demonstrate how collaboration success can be analyzed by exposing the processes that occurred during collaborative work.

\section{Process-Oriented Framework}

The current framework incorporates observable indicators of collaborations through two main strands: 1) Interpersonal dimension: how the social dynamics, as well as the individual research interests and contributions, shape group collaborations. 2) Project dimension: what specific project tasks and steps need to be completed in order to reach an outcome. Field knowledge, skills, and project commitment are integral to this dimension.

\footnotetext{
${ }^{2}$ CogNovo is an interdisciplinary doctoral training program jointly funded by the Marie Skłodowska Curie Actions and Plymouth University, comprising a network of diverse researchers from various disciplines, including Psychology, Computational Neuroscience, Robotics, Arts and Humanities.
} 
Of course, any given collaboration will have external conditions for success driven by institutional contexts (Mansilla et al., 2012). The conventions and expectations of both academic fields and funding organizations will inevitably contribute to the collaborative environment and both the interpersonal and project dimensions of any given project. The institutional context initiates, supports, and funds the collaborations and thus has a significant impact on the overall success of the collaborative endeavor. The examples described in the present paper include projects that were all completed within the same institutional contexts. As such, at the end of this paper we offer suggestions on how the process-oriented framework might be extended and adapted to take into account other institutional contexts.

\section{Interpersonal Dimension}

Within our process-oriented framework, the interpersonal aspect of a collaboration can arise in three different ways: 1) Mutual collaborations: every participant contributes to the collaboration equally and the contribution from different disciplines is weighted equally. Collaboration results in similar outcomes for all involved disciplines. 2) Assisted collaborations: the project is led by one discipline and collaborators from other disciplines assist by providing specific knowledge. Collaboration results in progress in the main discipline. 3) Emergent collaborations: these collaborations do not require a specific domain knowledge. Collaboration may occur on a primarily social or pragmatic level (e.g., departmental colleagues organizing a research seminar series) involving no particular discipline, or the collaboration may result in progress in a new (or emergent) discipline.

\section{Project Dimension}

All collaborations involve a project dimension, where certain tasks must be accomplished in order to achieve the desired outcomes. This dimension involves coordination between the participants' knowledge of domain(s) and relevant skills. For the projects evaluated within CogNovo, we identified the following primary steps: 1) Objective \& Research Question: formulating objectives and research questions;

2) Experiment: formulating and/or carrying out methods; 3) Analysis: formulating and/or carrying out analyses; 4) Communication: formulating and/or carrying out dissemination strategies to communicate collaboration outputs (e.g., writing papers). Even within the same context, these steps will have different importance and may even be skipped entirely depending on the implementation, aim, and success of the project. Importantly, although stages are described linearly here, the reiterative and adaptive transfer from one stage to another can be both dynamic and unpredictable. For example, it is likely that project objectives and research questions will be frequently revisited and revised at multiple times during a project lifecycle. 


\section{Process-Oriented Framework Scheme}

According to the process-oriented framework, each collaboration can be evaluated through the interpersonal and project dimensions on a point-based system. We propose that the four stages of a project should be measured independently. In the first stage, Objective \& Research Question, a research question is generated and a method is explored (and potentially tested). If the research question derives from and seeks to fill a gap in literature in two or more distinct domains, the collaboration can be seen as mutual. Instead, if the gap can be filled by applying knowledge or a method from one of the involved domains, the collaboration is assisted. Finally, if several researchers identify a potentially interesting topic outside of all their domains and create a question and method to answer it, the collaboration could be classified as emergent. Each project stage can be simultaneously mutual, assisted, and emergent to different degrees. An initial scoring of the project can be done in accordance with these three categories: we suggest that the sum of the categories for each row should be $100 \%$. For example, if a participant wants to express that a project was $2 / 3$ mutual, $1 / 3$ assisted, and not emergent at all, (s)he would score it as $67 \%$ mutual, $33 \%$ assisted, and $0 \%$ emergent.

The second stage, Experiment, includes any kind of data collection that contributes to answering the questions identified in the first stage. This type of data collection can be either grounded or contribute to several domains, and therefore it can be categorized as mutual. If the methodology is borrowed from one domain to address the data collection from a second one, this could be classified as assisted. Finally, if the method is taken from another line of work in which all participating researchers have only lay-people knowledge, this project would lay in the emergent collaboration category.

The third stage, Analysis, involves any kind of data processing that transforms the data collected in the previous stage into knowledge of some kind. The analysis may be driven by conventions prescribed by a single discipline. For example, in the sciences, both quantitative and qualitative methods could be applied at this stage, while in the humanities historical methods might be adopted, and in philosophy, conceptual analysis might be favored. It is also possible for the analysis to incorporate analytical procedures that combine several disciplinary approaches or that construct approaches that transcend traditional methods bound by a single discipline. Note that the rule of distributing $100 \%$ across the three columns also applies here.

At the final stage, Communication, results are communicated to others through various ways such as poster presentations, talks, papers, or even through chats and other forms of informal conversation. If a journal covers two or more research areas that the project is situated within, the communication can be seen as mutual. Instead, if the results are communicated at a specific conference but calling for support from a 
different domain, this communication would belong to the assisted category. Finally, communications such as open science, code repositories, or public engagements events, are considered primarily as belonging to the emergent category.

In parallel and independent of the measures collected for each stage, the importance (or weight) of the stage itself can also be rated. For a project that aims to generate new research questions, the main focus might be on the first and, to a lesser extent, on the fourth stage. For projects that focus on novel analysis of existing data, the second and third stage would receive more weight. The four stages cover the lifecycle of a project, thus the sum across all stages is $100 \%$. For example, if all stages have a similar weight, then they would each receive $25 \%$. An alternative to rating weights in hindsight is the amendment of stage weights based on the project aims.

Table 1 illustrates an example of this matrix system. In this case, the first step has almost $1 / 3$ of the overall weight $(30 \%)$. This project has no experimental aspect, thus the data collection method is not considered a valuable contribution by the collaborators. Likewise, the analysis plays only a small role (10\%). On the other hand, the communication of the results is rated as the most important part of this project and received a $60 \%$ weight in the overall rating. Based on this intuitive rating, an overall rating of $41 \%$ mutual, $15 \%$ assisted, and $44 \%$ emergent could be calculated for this project.

Table 1. Illustration of the evaluation matrix of proposed framework.

\begin{tabular}{|r|c|c|c|c|}
\cline { 3 - 5 } \multicolumn{1}{c|}{} & \multirow{2}{*}{ Weight } & \multicolumn{3}{c|}{ Classification } \\
\cline { 3 - 6 } & & Mutual & Assisted & Emergent \\
\hline Objective \& Research Questions & 30 & 70 & 30 & \\
\hline Experiment & & (N/A) & (N/A) & (N/A) \\
\hline Analysis & 10 & 20 & 60 & 20 \\
\hline Communication & 60 & 30 & 0 & 70 \\
\hline Overall rating & & $\mathbf{4 1}$ & $\mathbf{1 5}$ & $\mathbf{4 4}$ \\
\hline
\end{tabular}




\section{CogNovo Project Collaboration Examples}

\section{Example 1: “Bisensorial” (Hack the Brain 2016 Hackathon).}

Collaborators: Diego Maranan, Agi Haines, Jack McKay Fletcher, Sean Clarke, Kim Jensen, Ricardo Mutuberria

Disciplines: Design, Music, Cognitive Neuroscience, Computer Science, Psychology, Arts

Objective \& research questions: Ideate and prototype a "hack" based on the event theme, "Hacking yourself for better or for worse," that maximizes the skills of the participants and the resources available during the hackathon.

Result of design experiments: A working proof-of-concept of a wearable, neuroadaptive, vibroacoustic therapeutic device.

Communication: Presented at Hack the Brain 2016 event; exhibited at Off the Lip 2016 public engagement event, Bizarre Bazaar; exhibited at the Cognition Institute Conference; to be presented at the Ars Electronica STARTS event; discussed in PhD thesis (Maranan, 2017).

\section{Collaboration type:}

Table 2. Evaluation of Bisensorial Project.

\begin{tabular}{|r|c|c|c|c|}
\cline { 3 - 5 } \multicolumn{1}{c|}{} & \multirow{2}{*}{ Weight } & \multicolumn{3}{c|}{ Classification } \\
\cline { 3 - 5 } & & Mutual & Assisted & Emergent \\
\hline Objective \& Research Questions & 50 & 80 & 15 & 5 \\
\hline Experiment & 35 & 30 & 70 & 0 \\
\hline Analysis & & $(\mathrm{N} / \mathrm{A})$ & $(\mathrm{N} / \mathrm{A})$ & $(\mathrm{N} / \mathrm{A})$ \\
\hline Communication & 15 & 75 & 25 & 0 \\
\hline Overall rating & & $\mathbf{6 4 . 2 5}$ & $\mathbf{3 3}$ & $\mathbf{2 . 7 5}$ \\
\hline
\end{tabular}




\section{Example 2: BRAMZ (Because youR BrAin MatterZ)}

Disciplines: Psychology, Linguistics, Human-Computer Interaction

Collaborators: Ilaria Torre, Frank Loesche, Kathryn Francis, Raluca Briazu, David Bridges

Objective \& research questions: The main aim of this project was to develop personality measurements through games. Specifically, to build a mobile phone application in order to implement the games and collect data from experiment participants.

Experiment: Prototypical implementation during Computational Modelling workshop Analysis: No analysis was conducted.

Communication: Grant application for the "StudentshIP Enterprise Awards 2014."

\section{Collaboration type:}

Table 3. Evaluation of BRAMZ Project.

\begin{tabular}{|r|c|c|c|c|}
\cline { 3 - 5 } \multicolumn{1}{c|}{} & \multirow{2}{*}{ Weight } & \multicolumn{3}{c|}{ Classification } \\
\cline { 3 - 6 } & & Mutual & Assisted & Emergent \\
\hline Objective \& Research Questions & 30 & 70 & 30 & 0 \\
\hline Experiment & 10 & 20 & 60 & 20 \\
\hline Analysis & & (N/A) & (N/A) & (N/A) \\
\hline Communication & 60 & 30 & 0 & 70 \\
\hline Overall Rating & & $\mathbf{4 1}$ & $\mathbf{1 5}$ & $\mathbf{4 4}$ \\
\hline
\end{tabular}




\section{Example 3: Impasse in Conversations}

Disciplines: Linguistics, Psychology

Collaborators: Ilaria Torre, Frank Loesche

Objective \& research questions: Analyze creative problem solving in social interaction, in focusing in particular on how impasses are overcome in conversation.

Experiment: Choice of conversations, data collection not part of the project.

Analysis: Conversation analysis on freely available corpus of spontaneous conversations.

Communication: paper in Creativity: Theories-Research-Applications Journal (Torre \& Loesche, 2016); Poster presentation at UK Creativity 2017 Conference (Edinburgh)

\section{Collaboration type:}

Table 4. Evaluation of Impasse in Conversations Project.

\begin{tabular}{|r|c|c|c|c|}
\cline { 3 - 5 } \multicolumn{1}{c|}{} & \multirow{2}{*}{ Weight } & \multicolumn{3}{c|}{ Classification } \\
\cline { 3 - 5 } & & Mutual & Assisted & Emergent \\
\hline Objective \& Research Questions & 35 & 80 & 0 & 20 \\
\hline Experiment & 20 & 30 & 50 & 20 \\
\hline Analysis & 20 & 50 & 30 & 10 \\
\hline Communication & 25 & 70 & 0 & 30 \\
\hline Overall Rating & & $\mathbf{6 1 . 5}$ & $\mathbf{1 6}$ & $\mathbf{2 0 . 5}$ \\
\hline
\end{tabular}




\section{Example 4: Distorted Dimensions}

Disciplines/fields: Social Psychology, Cognitive Psychology, Philosophy, Art \& Design

Collaborators: Kathryn Francis, Agi Haines, Raluca Briazu

Objective \& research questions: Using moral psychology as a case study, we explored the importance of incorporating considerations from design research into the development of testing tools in the experimental sciences. We further considered how the process of "making" might be utilized as a collaborative tool, nurturing successful interdisciplinary endeavors.

Experiments/outputs/results: An interactive and life-like testing tool was constructed and incorporated in an existing moral decision-making experiment.

Communication: (a) Data were collected during an interactive installation with members of the public at OTLip16. (b) The data collected were incorporated into a scientific publication (Francis et al., 2017). (c) A conference paper exploring the use of "thinking through making" as an interdisciplinary collaborative tool was presented at OTLip17 (Francis et al., 2017.

\section{Collaboration type:}

Table 5. Evaluation of Distorted Dimensions Project.

\begin{tabular}{|r|c|c|c|c|}
\cline { 3 - 5 } \multicolumn{1}{c|}{} & \multirow{2}{*}{ Weight } & \multicolumn{3}{c|}{ Classification } \\
\cline { 3 - 5 } & & Mutual & Assisted & Emergent \\
\hline Objective \& Research Questions & 19 & 38 & 32 & 30 \\
\hline Experiment & 29 & 48 & 33 & 19 \\
\hline Analysis & 21 & 40 & 30 & 30 \\
\hline Communication & 31 & 42 & 20 & 38 \\
\hline Overall Rating & & $\mathbf{4 3}$ & $\mathbf{2 8}$ & $\mathbf{2 9}$ \\
\hline
\end{tabular}




\section{Conclusions}

Research contributions from large and diverse research groups play a key role in the solution of complex societal problems (Buanes \& Jentoft, 2009). Encouraging collaboration between various disciplines results in the sharing of domain-specific knowledge, but also in the emergence of new knowledge (De Stefano, Giordano, \& Vitale, 2011), thus providing novel solutions for unresolved age-old problems. Yet, what entails "successful" interdisciplinary collaboration has largely remained unclear (Huutoniemi et al., 2010).

To date, existing attempts to evaluate the impact of interdisciplinary research collaborations have sought to assess product-based outcomes, primarily considering coauthorship as a marker of success (Savanur \& Srikanth, 2010). Although these productbased approaches have allowed researchers to accurately quantify the impact of various disciplines within a collaboration (e.g., Groboljšek et al., 2014), they often overlook process-based markers of success. This is significant given that definitions of successful interdisciplinary collaborations encompass process-based considerations including mutual integration, cooperation, communication, and sharing (Aboelela et al., 2007).

In the current research, we formulated a novel framework for evaluating, as well as summarizing, research collaborations. By establishing a process-based framework, we have contributed to the literature by complementing the product-based approaches to evaluating collaborations. By generating a collaborative "fingerprint" for each project, the present process-oriented framework allows researchers to examine the interdisciplinary dynamics within a research group. This is significant for several reasons. Firstly, we can shed light on the "black box" that surrounds the understanding of collaborative processes (Kurtzberg \& Amabile, 2001). Secondly, we can use the process-based fingerprint to identify which group dynamics and which types of collaboration are more likely to succeed. This might be done by uniting our metric with product-based markers for success and/or measures of researcher satisfaction.

When considering the institutions, organizations, and funding bodies that support these collaborative endeavors, it is important to note that the collaboration examples described in the present paper were supported by the same institution and, as such, were fostered within the same organizational context. In order to extend our process-oriented framework, we suggest that future research should embrace the flexibility of the stages that we propose, adapting the metric to reflect the aims and constraints of their own organizational and institutional contexts.

Overall and through a detailed look at several collaboration examples that took place within the CogNovo project, we have developed a process-based approach for understanding both the interpersonal and project dimensions of interdisciplinary collaborations. Specifically, we have demonstrated that each collaboration is subject to different priorities and pressures. Thus, individual projects can display a unique 
combination of interpersonal dynamics and project tasks. Our process-oriented framework and evaluation matrix might be utilized not only to evaluate and provide building ingredients for successful interdisciplinary research collaborations, but also to quantify the impact of these collaborations beyond product-based metrics.

\section{Acknowledgements}

We would like to Klara Łucznik and Joan ten Hoonte for their comments on an earlier version of this paper and all participants at Off the Lip 2017: CogNovo Colloquium on Experiences and Applications of Cognitive Innovation for the fruitful discussion.

CogNovo and this study are jointly funded by Plymouth University and the Marie Skłodowska-Curie actions (MSCA) program (FP7-PEOPLE-2013-ITN-604764).

\section{References}

Aboelela, S. W., Larson, E., Bakken, S., Carrasquillo, O., Formicola, A., Glied, S. A., . . Gebbie, K. M. (2007). Defining interdisciplinary research: Conclusions from a critical review of the literature. Health Services Research, 42(1p1), 329-346. doi:10.1111/j.1475-6773.2006.00621.x

Ajiferuke, I., Burell, Q., \& Tague, J. (1988). Collaborative coefficient: A single measure of the degree of collaboration in research. Scientometrics, 14(5-6), 421-433. doi:doi.org/10.1007/BF02017100

Bukvova, H. (2010). Studying research collaboration: A literature review. Sprouts: Working Papers on Information Systems, 10(3). Retrieved from http://sprouts.aisnet.org/10-3

Bruce, A., Lyall, C., Tait, J., \& Williams, R. (2004). Interdisciplinary integration in europe: The case of the Fifth Framework Programme. Futures, 36(4), 457-470. doi:10.1016/j.futures.2003.10.003

Buanes, A., \& Jentoft, S. (2009). Building bridges: Institutional perspectives on interdisciplinarity. Futures, 41(7), 446-454.

Callard, F., \& Fitzgerald, D. (2015). Rethinking interdisciplinarity across the social sciences and neurosciences. Basingstoke, UK: Palgrave Macmillan.

Choi, B. C. K., \& Pak, A. W. P. (2006). Multidisciplinarity, interdisciplinarity and transdisciplinarity in health research, services, ducation and policy: 1 . Definitions, objectives, and evidence of effectiveness. Clinical and Investigative Medicine, 29(6), 351-364.

De Stefano, D., Giordano, G., \& Vitale, M. P. (2011). Issues in the analysis of co-authorship networks. Quality \& Quantity, 45(5), 1091-1107. doi:10.1007/s11135-011-9493-2

Francis, K. B., Haines, A., Briazu, R. A. (2017). Thinkering through experiments: Nurturing transdisciplinary approaches to the design of testing tools. AVANT, 8(Special Issue), 107-119. doi:10.26913/80s02017.0111.0011

Francis, K. B., Terbeck, S., Briazu, R. A., Haines, A., Gummerum, M., Ganis, G., \& Howard, I. S. (2017). Simulating moral actions: An investigation of personal force in virtual moral dilemmas. Scientific Reports, 7(1). doi:10.1038/s41598-017-13909-9 
Groboljšek, B., Ferligoj, A., Mali, F., Kronegger, L., \& Iglič, H. (2014). The role and significance of scientific collaboration for the new emerging sciences: The case of Slovenia. Teorija in praksa, 51(5), 866-885.

Huutoniemi, K., Klein, J. T., Bruun, H., \& Hukkinen, J. (2010). Analyzing interdisciplinarity: Typology and indicators. Research Policy, 39(1), 79-88. doi:10.1016/j.respol.2009.09.011

Katz, J. S., \& Martin, B. R. (1997). What is research collaboration? Research Policy, 26(1), 1-18. https://doi.org/10.1016/S0048-7333(96)00917-1

Klein, J. T. (2008). Evaluation of interdisciplinary and transdisciplinary research: A literature review. American Journal of Preventive Medicine, 35(2, Supplement), S116-S123. doi:10.1016/j.amepre.2008.05.010

Kurtzberg, T. R., \& Amabile, T. M. (2001). From Guilford to creative synergy: Opening the black box of team-level creativity. Creativity Research Journal, 13(3-4), 285-294. doi:10.1207/S15326934CRJ1334_06

Lawrence, R. J. (2010). Deciphering interdisciplinary and transdisciplinary contributions. Transdisciplinary Journal of Engineering \& Science, 1(1), 125-130.

Larivière, V., Gingras, Y., Sugimoto, C. R., \& Tsou, A. (2014). Team size matters: Collaboration and scientific impact since 1900. Journal of the Association for Information Science \& Technology, 66(7), 1323-1332.

Mansilla, V. B., Lamont, M., \& Kyoko Sato, K. (2012, February). The contributions of shared socioemotional-cognitive platforms to interdisciplinary synthesis. Paper presented at the 4S Annual Meeting, Vancouver, Canada.

Maranan, D. S. (2017). Haplós: Towards Technologies for and Applications of Somaesthetics (Doctoral dissertation). Plymouth University, Plymouth, UK. Retrieved from http://hdl.handle.net/10026.1/10170

Maranan, D. S., Loesche, F., \& Denham, S. L. (2015). CogNovo: Cognitive innovation for technological, artistic, and social domains. In Proceedings of the 21st International Symposium on Electronic Arts. Retrieved from http://hdl.handle.net/10026.1/5076

Patel, H., Pettitt, M., \& Wilson, J. R. (2012). Factors of collaborative working: A framework for a collaboration model. Applied Ergonomics, 43(1), 1-26. doi:10.1016/j.apergo.2011.04.009

Savanur, K., \& Srikanth, R. (2010). Modified collaborative coefficient: A new measure for quantifying the degree of research collaboration. Scientometrics, 84(2), 365-371. doi:10.1007/s11192-009-0100-4

Subramanyam, K. (1983). Bibliometric studies of research collaboration: A review. Information Scientist, 6(1), 33-38. doi:10.1177/016555158300600105

Torre, I., \& Loesche, F. (2016). Overcoming impasses in conversations: A creative business, Creativity: Theories - Research - Applications, 3(2), 244-260. doi:10.1515/ctra-2016-0

van Rijnsoever, F. J., \& Hessels, L. K. (2011). Factors associated with disciplinary and interdisciplinary research collaboration. Research Policy, 40(3), 463-472. doi:10.1016/j.respol.2010.11.001 